\title{
SAGE: A Monte Carlo Simulation Framework for Experiments with Germanium Detectors
}

\author{
Ze She ${ }^{a}$ Hao Ma ${ }^{a}$ Weihe Zeng ${ }^{a}$ Wenhan Dai $^{a}$ Xinping Geng ${ }^{a}$ Ofoq Normahmedov ${ }^{a}$ \\ Jingzhe Yang ${ }^{a}$ Zhi Zeng ${ }^{a, 1}$ Qian Yue $^{a}$ Jianping Cheng ${ }^{a, b}$ Junli Li ${ }^{a}$ \\ ${ }^{a}$ Key Laboratory of Particle and Radiation Imaging (Ministry of Education) and Department of Engineering \\ Physics, Tsinghua University, Beijing 100084 \\ ${ }^{b}$ College of Nuclear Science and Technology, Beijing Normal University, Beijing 100875 \\ E-mail: zengzhi@mail.tsinghua.edu.cn
}

\begin{abstract}
A Geant4-based simulation framework for rare event searching experiments with germanium detectors named SAGE is presented with details. It is designed for simulating, assessing background distribution, and investigating the response of the germanium detectors. The SAGE framework incorporates its experiment-specific geometries and custom attributes, including the event generators, physics lists and output format, to satisfy various simulation objectives. Its docker image has been prepared for virtualizing and distributing the SAGE framework. Deployment of a Geant4-based simulation will be convenient under this docker image. The implemented geometries include p-type point contact and broad energy germanium detectors with environmental surroundings, and these hierarchical geometries can be easily extended. Users select these custom attributes via the JSON configuration files. The aforementioned attributes satisfy the simulation demands, and make SAGE a generic and powerful simulation framework for the CDEX experiment.
\end{abstract}

KeYwORDs: Detector modelling and simulations I

${ }^{1}$ Corresponding author. 


\section{Contents}

1 Introduction 1

2 Structure of SAGE 2

$\begin{array}{llr}2.1 \text { Geometries } & 2\end{array}$

2.2 Event generator 3

2.3 Physics Lists 3

$\begin{array}{lll}2.4 & \text { Output } & 5\end{array}$

3 Use Case 5

4 Summary 9

\section{Introduction}

The background models are essential for conceptual or technical design and data analysis of rare event searching experiments. The background simulation and assessment raises the substantive requirements for the experimental design and material selections, and ultimately promotes the construction of the experimental setup [1,2]. While in the statistical analysis, a more precise background model ascertains a more convincing and robust conclusion[3-7]. For rare event searching experiments, it is necessary to have a unified and convincing simulation framework for establishing their background models. The simulation works can be conducted more efficiently within collaboration under these unified simulation frameworks [8].

SAGE (abbreviation for Simulation and Analysis for Germanium Experiments) is based on the Geant 4 toolkit [9-11] and mainly developed by the China Dark matter EXperiment (CDEX) [12-15], which is located in hall C of the China Jinping Laboratory phase-II (CJPL-II) [16]. It is designed for simulating the background contributions for the ultra-low background germanium experiments such as dark matter (DM) searching, neutrinoless double beta decay $(0 v \beta \beta)$ of ${ }^{76} \mathrm{Ge}$, as well as material screening by germanium gamma-ray spectrometers. Currently, SAGE offers flexible interfaces for different experimental configurations. It satisfies the need to accelerate the realizations and remove the challenges of simulations, such as knowledge of $\mathrm{C}++$ and achieves consensus on the preset parameters in Geant4, so that the simulation works can become efficient, robust and reproducible. SAGE allows its users to run a variety of simulations without writing any $\mathrm{C}++$ code. Its user-friendly functionalities and reliable preset parameters in the simulation workflow make SAGE a generic, powerful and user-friendly simulation framework.

This paper describes the software framework and custom features of SAGE. Its hierarchy and the detailed structures are discussed in Section 2. A configuration file is provided in Section 3, presenting as a manual of SAGE. The last section summarizes all features and use of SAGE. 


\section{Structure of SAGE}

SAGE has been compiled and run with versions 10.6 and 10.7 of Geant4. The modular design makes SAGE a flexible and extensible simulation framework. Its hierarchy is depicted as shown in figure 1, and the following parts detail the implementation of each module. The SAGESimManager module supervises the simulation flow and enables the multi-threading capabilities provided by Geant4.

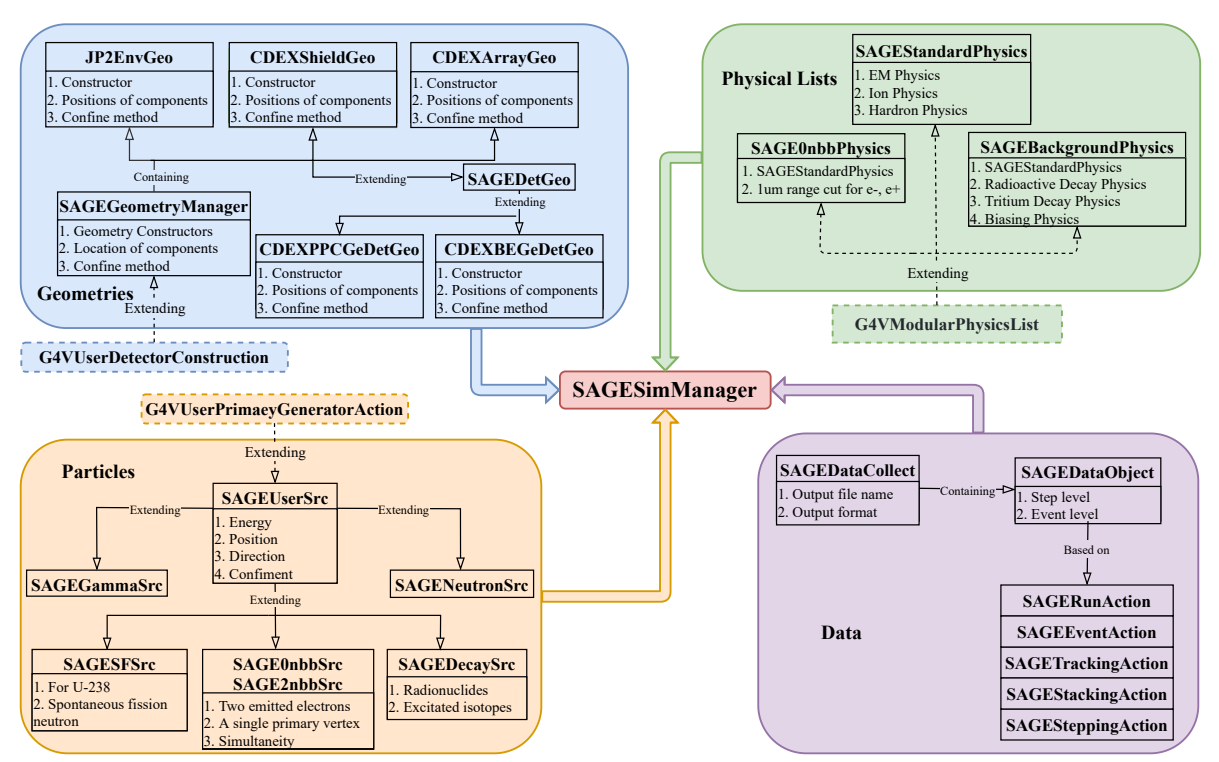

Figure 1: The software structure of SAGE follows the trace of Geant4 simulation. The JSON configuration file delivers the user-defined parameters to the SAGE simulation manager while this simulation manager controls of subsequent workflow.

\subsection{Geometries}

This module is responsible for constructing the experimental geometries, and the Geant 4 intrinsic geometry description classes support it. SAGE provides a geometry manager for organizing hierarchical geometries and an abstract class SAGEDetGeo for deriving different kinds of germanium detectors. Custom geometries should be registered with the geometry manager.

Some experiments with germanium detectors are searching for rare events, including dark matter and $0 v \beta \beta$ decay of ${ }^{76} \mathrm{Ge}$, such as SuperCDMS [17], Gerda [18], CDEX [12], EDELWEISS [19]. These experiments are conducted with the massive germanium detector as a detector array for enlarging their exposure. Taken the CDEX experiment as an example, SAGE can be used for assessing the background distributions for these experiments. Its germanium detector array is located in the stainless-steel cryostat filled with liquid nitrogen, as shown in figure 2. Therefore, this detector array can be dynamically enlarged, and the existing detector unit geometric classes can be reusable provided that they can be contained. The germanium detectors are also highly used as gamma-ray spectrometers, such as GeTHU [20], Gator [21], BUGS [22]. SAGE assists in the construction of their background models and the simulation of their detection efficiencies. SAGE has been used in the simulation for GeTHU whose geometry is shown in figure 2. 

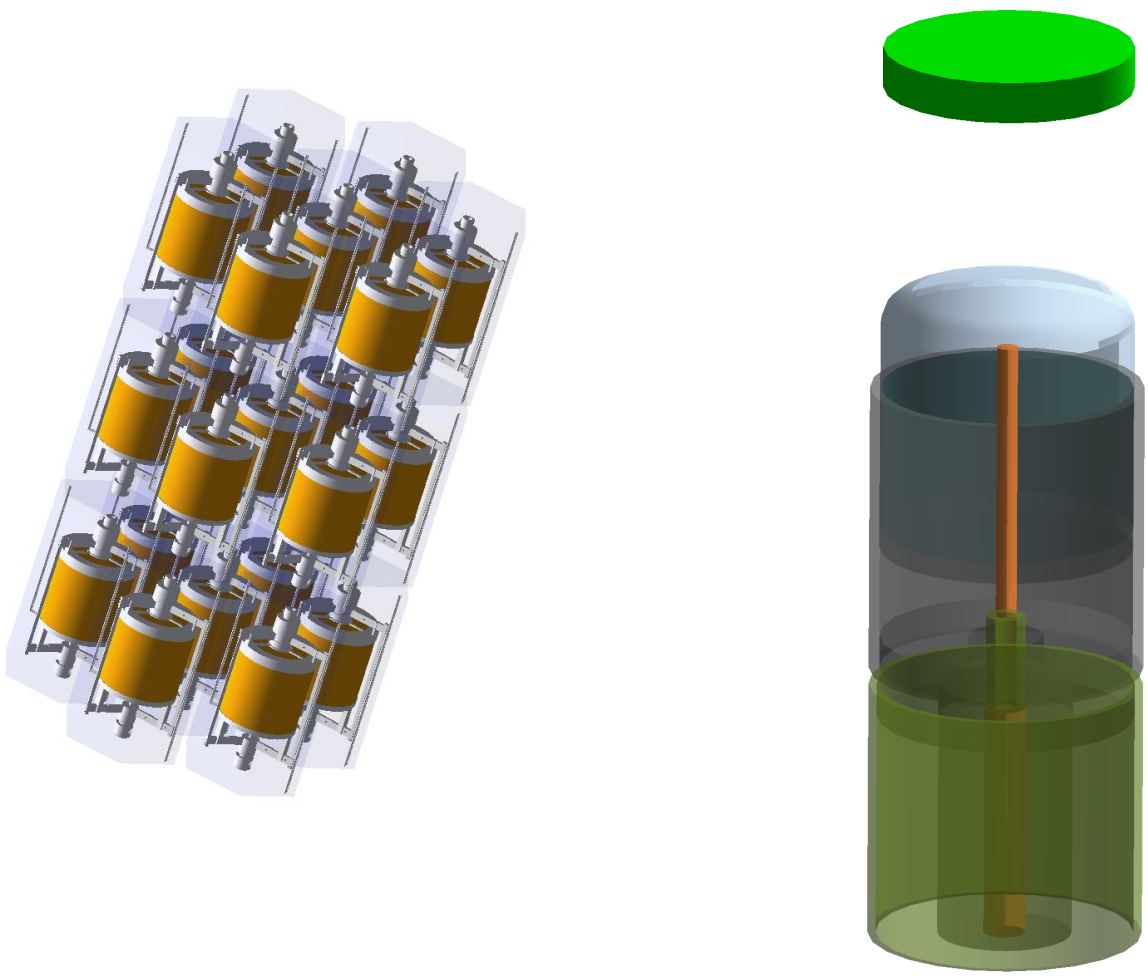

Figure 2: The geometric structures for germanium detectors of CDEX-100 (left) and GeTHU (right).

\subsection{Event generator}

Customized event generators originate from an abstract class SAGEUserSrc which is based on the G4SingleParticleSource. These customized event generators produce particles including neutron, electron, gamma, radionuclides, $0 v \beta \beta$ and $2 v \beta \beta$ isotope source [23]. Although the $0 v \beta \beta$ and $2 v \beta \beta$ sources cannot be generated with G4SingleParticleSource directly, two electrons are attached to the primary vertex to generate two electrons simultaneously by an embedded algorithm. In addition to the point, surface and volume sources, we attach the CDEX geometric volumes to these generators through a member function (named ConfinePosition) of the abstract class SAGEUserSrc to facilitate the sampling strategies.

\subsection{Physics Lists}

The physics list module is constructed and optimized for germanium detectors by capitalizing the Geant4 intrinsic physics lists. It was designed according to the suggestions of the Geant 4 team and the related examples in the Geant 4 package. Three available physics lists SAGEStandardPhysics, SAGEOnbbPhysics and SAGEBackgroundPhysics are optimized for different simulation projects. Among these physics lists, the SAGEStandardPhysics is the simplest and most efficient one. The SAGEStandardPhysics physics list consists of modular physics lists including G4DecayPhysics, G4EmStandardPhysics_option3, G4EmExtraPhysics, G4HadronPhysicsFTFP_BERT, G4HadronElasticPhysics, G4StoppingPhysics, G4IonPhysics. 
These modular physics lists satisfy the simulation requirements of germanium detectors in most cases, and SAGEStandardPhysics is used by default, but more requirements are raised in some instances. Based on SAGEStandardPhysics, the other two physics lists integrate more features for their simulation aims. SAGEOnbbPhysics is tailored for tracking electrons with a smaller step limit of electrons ( $1 \mu \mathrm{m}$ rather than $1 \mathrm{~mm}$ default value) with about 15 times larger than CPU consuming time. With an 1- $\mu \mathrm{m}$ step limit for an electron, its track will be terminated when its energy is less than $10 \mathrm{keV}$, but an 1-mm step limit terminates for $800 \mathrm{keV}$. It can be applied to investigate the characteristics of pulses generated from the charge collections or the pre-amplifier. SAGEBackgroundPhysics is intended for the ambient and other intrinsic background contributions of germanium detectors. SAGEBackgroundPhysics can be used to establish background models and assess the background indices for experiments. SAGEBackgroundPhysics has two additional features.

(1) The $\beta$-decay of ${ }^{3} \mathrm{H}$.

${ }^{3} \mathrm{H}$ is the product of cosmogenic activation inside the germanium crystal, decays and then emits an electron with a maximum energy of $18.6 \mathrm{keV}$ and an anti-neutrino. It is treated as a stable isotope in Geant4, however. Users must calculate the energy spectrum of ${ }^{3} \mathrm{H} \beta$-decay before simulations and sample the incident electrons in deference to their energy distribution. SAGE instantiates a relevant physics sub-list named SAGETritiumPhysics to make ${ }^{3} \mathrm{H}$ decay automatically in Geant4.

(2) Biasing sampling

The second one is a generic biasing physics sub-list. When a radioactive source locates far from the detector (a remote radioactive source) or the cross-sections of certain interactions are too small to obtain the statistically significant results, such as simulating the gammarays background contribution decreased by shielding, simulation with $2^{31}$ incident particles (the maximum value of a single run in Geant4) probably cannot get an acceptable result unless simulating multiple runs. However, multiple runs will consume more computation time, and the results may still not be meaningful within reasonable simulation events under certain circumstances. SAGE has integrated two kinds of biasing techniques inside this generic biasing sub-list to alleviate these dilemmas. Russian roulette and split strategy [24], commonly used in deep penetration problems, is embedded in SAGE to tackle the simulation issue when the remote sources are distributed outside shielding. This method is similar to the Geant 4 example (extended/biasing/GBO3) other than the necessary concatenations with other SAGE modules. The shielding should be divided into multiple layers depending on their locations, and particles will participate in the biasing games when they occur at the boundaries of layers. When a particle in the direction of detectors occurs at the boundaries of layers, its track will be cloned multiple times. And then, these tracks will be simulated separately with the weight divided by the number of tracks. Otherwise, it might be killed according to the surviving probability $\mathcal{P}$ defined by users. If a particle survives Russian roulette, its track will have a weight increased by a factor of $1 / \mathcal{P}$. More computation power is assigned to particles with greater possibilities of reaching the germanium detectors through the split and kill method. When considering the interactions with tiny cross-sections such as neutron 
originated from $(\alpha, n)$ interactions, their cross-sections should be artificially increased before simulation; otherwise, they will not be observed. Although a tiny cross-section weakens the potential for neutron $(\alpha, n)$ interactions, the enormous mass of surrounding construction materials, such as concrete or stainless steel cryostat, makes neutrons detectable in the ultralow background experiments. To make them also observable in the simulations within limited events, SAGE adopts the change cross-section biasing method [25] as a variant of the strategy described in extended/biasing/GB01. When calculating the mean free path, the contributions from selected interactions are multiplied by a transformation factor determined by users. Then more events interact via the selected interactions, and more computation power is assigned to these interactions. Further discussion on this method can be found in [26].

\subsection{Output}

SAGE instantiates a SAGEDataCollect object to gather the interaction and trajectory information as particles interact with materials step by step. SAGE provides two intrinsic analysis levels with the SAGEDataObject object. The former one is called the "event" level, which analyzes all steps in a single event to get its energy deposition (grouped by the interaction mediators, such as electrons, neutrons, other particles), track length, living time(the time since it is created) and weight.

In simulations of radioactive decay chains, SAGE adopts the separation time of $10 \mu s$, roughly the detector response time, to distinguish radiogenic $\alpha, \beta$ and $\gamma$ rays from different isotopes in a single radioactive chain while their energies are by default recorded in a single event by Geant 4 . If the global time of the current step is greater than $10 \mu \mathrm{s}$, the simulation manager will reset its global time and record all interactions before the current step. The latter one named "step" level, recording and dumping all step information for debugging or pulse shape discrimination, contains the PDG encoding of particles, energy deposition, kinetic energy, interaction length, living time, weight, velocity direction, position and weight. Both levels have three output formats, i.e., *.csv, *.root or *.h5, for further analysis with the specified analysis tools.

\section{Use Case}

Users can adjust the free parameters in the configuration files. SAGE is assisted by a third-party module of JSON parser [27] to convert the configuration files into a dictionary data structure. A set of applicable JSON configuration files are provided in the SAGE software package as the user guides. The configuration files are arranged in six parts concerning the free simulation ingredients as follows:

(1) Mode: Two modes are provided. The "Simulation" mode means conducting simulation more efficiently with command lines, while the "Visualization" mode is helpful when you are curious about or debugging the simulation geometries. The visualization mode depends on the Geant4 visualization setup and is also named interactive mode due to its instant response to the manual input messenger command.

(2) Physics: There exist three physics lists related to different simulation goals. The "background" physics list contains the radioactive decay processes while the "0nbb" physics list has a smaller step limit for electrons. The "standard" physics list can be used if time efficiency is sensitive. 
(3) Array: Constructional parameters of the germanium detector arrays, containing the number of detectors, the distance between detectors, the type and material ("natural" or "enriched" germanium) of germanium detectors.

(4) Particle: The information of incident particles, such as particle name, energy, momentum direction, position and the number of incident particles.

(5) Biasing: Status and parameters of biasing techniques. In a normal case, biasing is disabled by default.

(6) Output: The output file name and the output level ("event" or "step" data object).

All of these properties have their default values for removing redundant parameters. For example, the biasing methods are disabled in default, so they can be neglected if not necessary.

SAGE has been compiled and linked into a single executable application together with a set of instructive configuration files. To distribute it more conveniently, SAGE and Geant4 have been wrapped inside a single docker image. The docker container compiles the Geant4.10.7 and SAGE, so users run SAGE directly after pulling. With a docker container, it is much easier for users to customize, distribute and use SAGE. The structure of this container is shown in figure 3. In addition, the docker file is also provided to build images for users' demands.

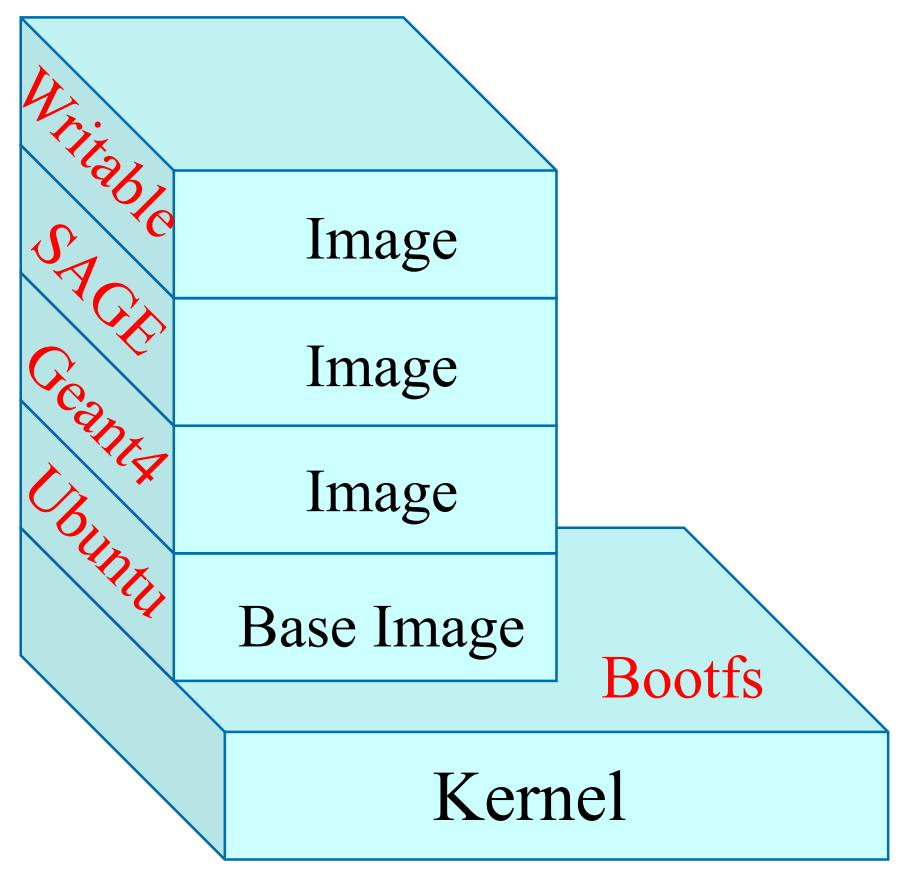

Figure 3: Docker image for SAGE.

A graphical user interface (GUI) with Electron, a framework for creating native applications with web technologies, is developed to make it more user-friendly to newbies. The GUI takes charge of generating configuration files and provides a shortcut to start the simulation task with a single click. The parameters shown in the GUI can be modified to satisfy the keywords defined by users. For GeTHU, Its GUI is shown in figure 4. The interface is divided into three categories. 
The file name should be written in the card named Project name. The geometry of samples can be selected in the card named Geometry Parameters which is consisted of several arguments including shape (cylinder or cuboid), dimensions of shapes, location and materials. The energy regions or nuclides of interest are added and stored with a list shown on the right via the card named Particle Source Parameters. The number of simulated events is also included in this card. SAGE will be executed with the configuration generated by this GUI tool by clicking the start button at the right bottom corner.

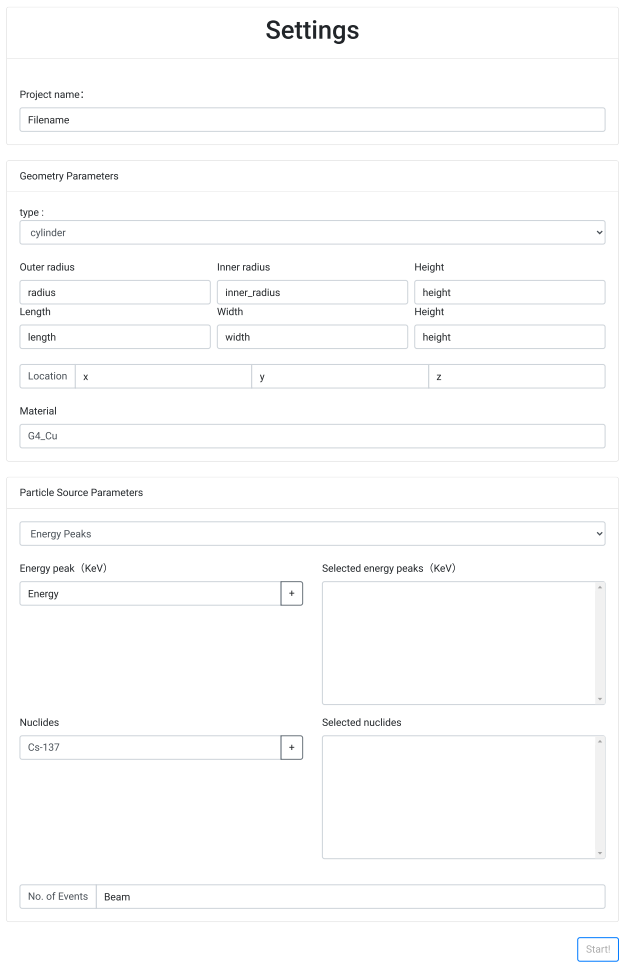

Figure 4: The default GUI of SAGE designed for GeTHU gamma-ray spectrometer.

SAGE has been widely used to simulate energy spectra in germanium detectors induced by radioactive samples and background sources, e. g. cosmogenic and primordial radionuclides for GeTHU [20] and CDEX experiment [28]. For example, the radionuclides ${ }^{60} \mathrm{Co}$ in the germanium crystal for the CDEX-100 experiment can be simulated with the configuration below:

\{

"Mode" : "Simulation",

"Physics" : "Background",

"Array" :

\{

"HeightGap " : 0 ,

"RadiusGap " : 0 ,

"HeightN" : 5,

"RadiusN" : 5, 


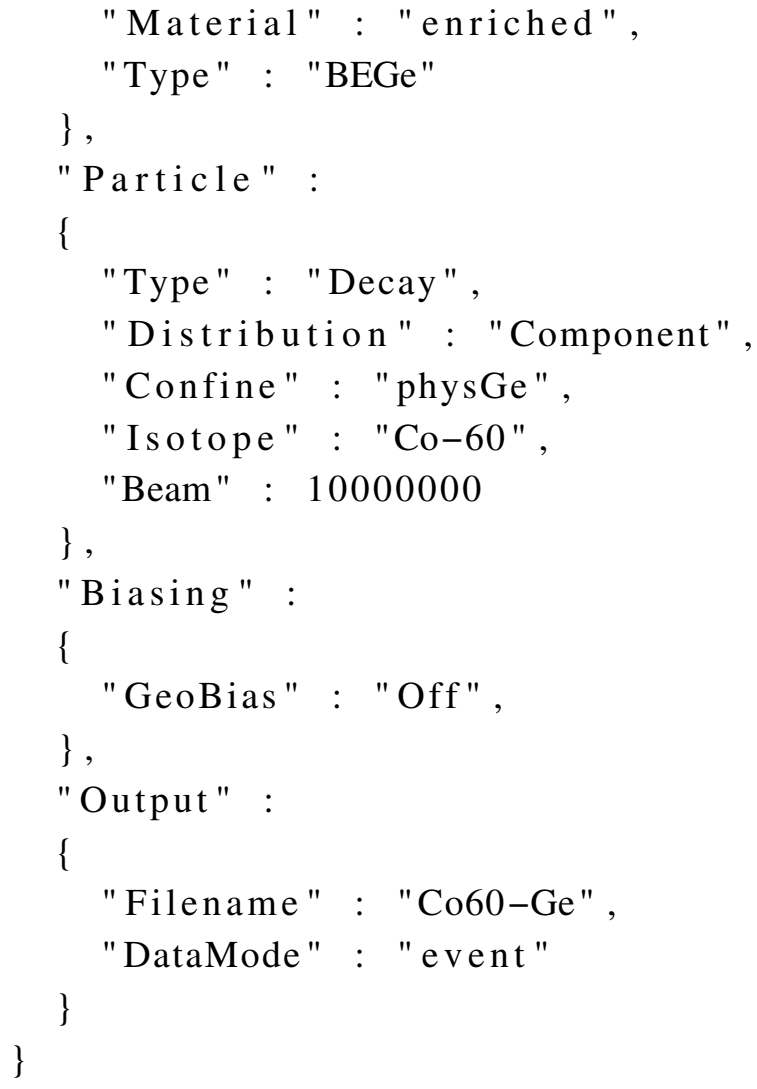

When we execute the SAGE with the configuration file above, we will get all simulation events in the Co60-Ge_nt_data.csv. Based on this output, the expected energy spectrum can be reconstructed, as shown in figure 5. Three peaks shown in figure 5 are two gamma-ray peaks (1.173 and $1.332 \mathrm{MeV})$ of ${ }^{60} \mathrm{Co}$ and the sum peak of these two gamma rays, respectively. Besides, the Compton edges of two gamma-ray peaks lead to an unusual increasing between 0.6 and $1.1 \mathrm{MeV}$ energy region. In the case of intrinsic background simulation inside the germanium crystal, the contributions of $\beta$-decay are also visible in the low-energy region of the energy spectrum. The events recording the energy deposition of both $\beta$-decay and subsequent gamma rays contribute to the non-gaussian shape of the gamma-ray peaks.

SAGE is validated by the calculations on the cross-sections of proton activations. Five natural lead samples were irradiated by different energies (40,70,100, 400 and $800 \mathrm{MeV})$ protons from 2000 to 2002 [29] and were measured with GeTHU in September 2020 at CJPL. SAGE is used to simulate their energy spectra of proton-induced radioactive isotopes, and then the measured energy spectrum is decomposed into these simulated energy spectra. The cross-sections are calculated based on the decomposed spectra and compatible with previous results [30].

Thanks to its modular design, SAGE can be widely used and extended in various experiments with different physics goals. For instance, the background spectra can be simulated after implementing the experiment-specific geometries inherited from the abstract class SAGEDetGeo. It assists the experimental setup design, assesses the background level, investigates detector response and estimates the projected sensitivities for experiments with germanium detectors. 


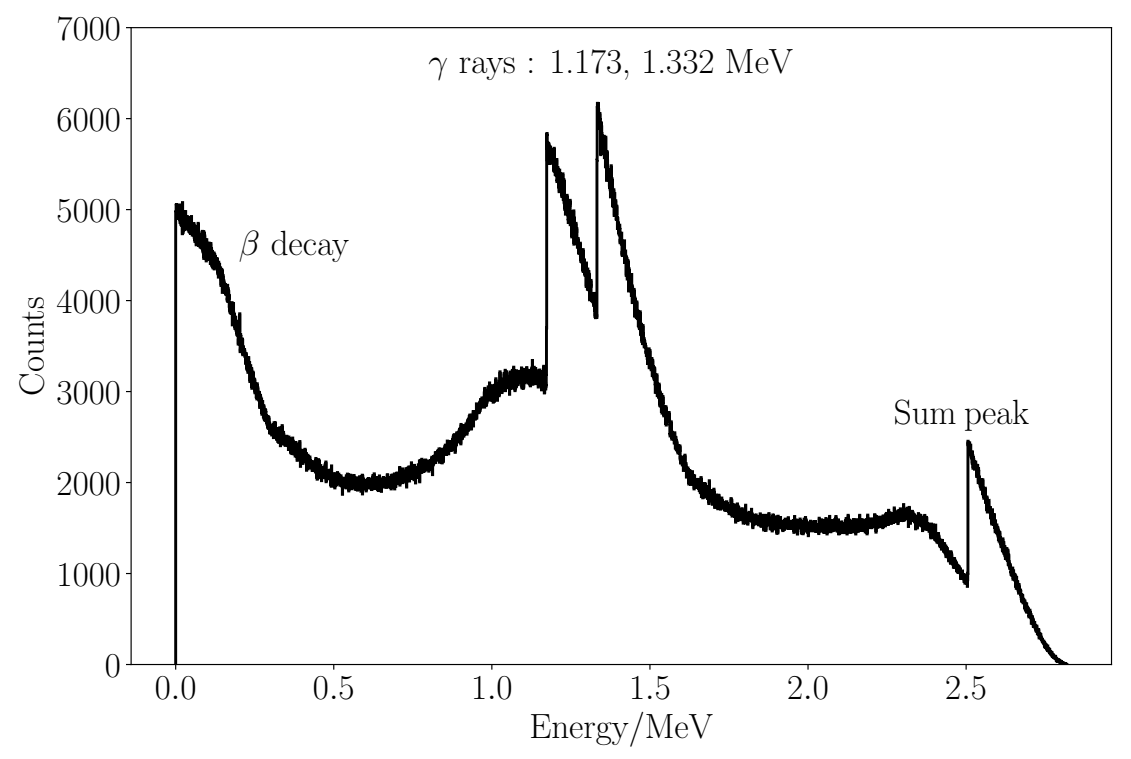

Figure 5: The simulated energy spectrum of cosmogenic ${ }^{60} \mathrm{Co}$ in germanium crystal.

\section{Summary}

In this work, we describe the simulation framework of SAGE and its customized features to simulate the particle trajectories in experiments using germanium detectors. Its configuration files are written in JSON format, and flexible parameters in the simulation workflow can be determined. SAGE has integrated the physics list for ${ }^{3} \mathrm{H}$ decay and biasing sampling techniques. All customized attributes make SAGE a unified, user-friendly, robust and reproducible simulation framework for germanium experiments and SAGE widely used in the CDEX collaboration. Currently, additional features are under investigation as follows to broaden its application.

- More geometries

- Statistical methods for further data analysis

- Pulse shape simulations and discriminations

Although SAGE is designed for experiments using germanium detectors, its implemented modules, except experiment-specific geometries, can be widely used in other rare event searching experiments having different detection techniques, which makes SAGE a more powerful and general simulation framework.

\section{Acknowledgments}

This work was supported by the National Key Research and Development Program of China (Grant No.2017YFA0402201), the National Natural Science Foundation of China (Grants No.U1865205 \& 11725522 \& 11675088), and Tsinghua University Initiative Scientific Research Program (No.2019050007). 


\section{References}

[1] J. L. Ma et al., Study on Cosmogenic Activation in Germanium Detectors for Future Tonne-scale CDEX Experiment, Sci. China Phys. Mech. 62 (2019) 11011.

[2] E. Aprile et al., Material Radioassay and Selection for the XENONIT Dark Matter Experiment, Eur. Phys. J. C 77 (2017) 890.

[3] M. Agostini et al., Modeling of Gerda Phase II data, J. High Energy Phys. 3 (2020) 139.

[4] C. Cuesta et al., Background Model for the Majorana Demonstrator, Phys. Proc. 61 (2015) 821.

[5] XENON collaboration, XENONIT Dark Matter Data Analysis: Signal Reconstruction, Calibration, and Event Selection, Phys. Rev. D 100 (2019) 052014.

[6] R. Agnese, Maximum Likelihood Analysis of Low Energy CDMS II Germanium Data, Phys. Rev. D 91 (2015) 052021.

[7] V. A. Kudryavtsev and J. L. Orrell, Expected Background in the LZ Experiment, AIP Conf. Proc. 1672 (2015) 060003.

[8] M. Boswell et al., MaGe-a Geant4-Based Monte Carlo Application Framework for Low-Background Germanium Experiments, IEEE T. Nucl. Sci. 58 (2011) 1212.

[9] S. Agostinelli et al., Geant4-A Simulation Toolkit, Nucl. Instrum. Meth. A 506 (2003) 250.

[10] J. Allison et al., Geant4 Developments and Applications, IEEE T. Nucl. Sci. 53 (2006) 270.

[11] J. Allison et al., Recent Developments in Geant4, Nucl. Instrum. Meth. A 835 (2016) 186.

[12] CDEX collaboration, Limits on Light Weakly Interacting Massive Particles from the First $102.8 \mathrm{~kg} \times$ day Data of the CDEX-10 Experiment, Phys. Rev. Lett. 120 (2018) 241301.

[13] CDEX collaboration, Search for Light Weakly-Interacting-Massive-Particle Dark Matter by Annual Modulation Analysis with a Point-Contact Germanium Detector at the China Jinping Underground Laboratory, Phys. Rev. Lett. 123 (2019) 221301.

[14] CDEX collaboration, Constraints on Spin-Independent Nucleus Scattering with sub-GeV Weakly Interacting Massive Particle Dark Matter from the CDEX-1B Experiment at the China Jinping Underground Laboratory, Phys. Rev. Lett. 123 (2019) 161301.

[15] CDEX collaboration, Direct Detection Constraints on Dark Photons with the CDEX-10 Experiment at the China Jinping Underground Laboratory, Phys. Rev. Lett. 124 (2020) 111301.

[16] J. P. Cheng et al., The China Jinping Underground Laboratory and Its Early Science, Annu. Rev. Nucl. Part. Sci. 67 (2017) 231.

[17] D. W. Amaral et al., Constraints on low-mass, relic dark matter candidates from a surface-operated SuperCDMS single-charge sensitive detector, Phys. Rev. D 102 (2020) 091101.

[18] GERDA collaboration, Final Results of GERDA on the Search for Neutrinoless Double- $\beta$ Decay, Phys. Rev. Lett. 125 (2020) 252502.

[19] EDELWEISS collaboration, First Germanium-Based Constraints on Sub-MeV Dark Matter with the EDELWEISS Experiment, Phys. Rev. Lett. 125 (2020) 141301.

[20] Z. Zeng et al., The Characteristics of a Low Background Germanium Gamma Ray Spectrometer at China JinPing underground laboratory, Appl. Radiat. Isotopes 91 (2014) 165.

[21] L. Baudis, A. D. Ferella, A. Askin et al., Gator: a low-background counting facility at the Gran Sasso Underground Laboratory, J. Instrum. 6 (2011) P08010. 
[22] P. R. Scovell, E. Meehan, H. M. Araújo et al., Low-background gamma spectroscopy at the boulby underground laboratory, Astropart. Phys. 97 (2017) 160.

[23] V. I. Tretyak and Y. G. Zdesenko, Tables of Double Beta Decay Data, Atom. Data Nucl. Data 61 (1995) 43.

[24] Monte Carlo integrations II: Improving efficiency, in Physically Based Rendering, M. Pharr and G. Humphreys, eds., (Burlington), pp. 663 - 718, Morgan Kaufmann, (2004).

[25] C. P. Robert and G. Casella, Monte Carlo Integration, in Monte Carlo Statistical Methods, (New York, NY), pp. 79-122, Springer New York, (2004).

[26] E. Mendoza et al., Neutron Production Induced by $\alpha$-Decay with Geant4, Nucl. Instrum. Meth. 960 (2020) 163659.

[27] “nlohmann/json." http://github.com/nlohmann/json.

[28] H. Ma et al., In-situ Gamma-ray Background Measurements for Next Generation CDEX Experiment in the China Jinping Underground Laboratory, Astropart. Phys. 128 (2021) 102560.

[29] Y. E. Titarenko et al., Excitation functions of product nuclei from 40 to $2600 \mathrm{MeV}$ proton-irradiated 206,207,208,natPb and 209Bi, Nucl. Instrum. Meth. A 562 (2006) 801.

[30] Y. Titarenko et al., $208,207,206, n a t ~ P b(p, x){ }^{207} B i$ and ${ }^{209} B i(p, x)^{207} B i$ excitation functions in the energy range of 0.04 - $2.6 \mathrm{GeV}$, Nuclear Instruments and Methods in Physics Research Section A: Accelerators, Spectrometers, Detectors and Associated Equipment 984 (2020) 164635. 